TTP $00-21$

September 22, 2000

\title{
Radiatively corrected shape function for inclusive heavy hadron decays
}

\author{
Thomas Mannel| and Stefan Recksiegelf \\ Institut für Theoretische Teilchenphysik, D-76128 Karlsruhe, Germany.
}

\begin{abstract}
We discuss the non-perturbative and the radiative corrections to inclusive $B$ decays from the point of view known from QED corrections to high energy $e^{+} e^{-}$processes. Here the leading contributions can be implemented through the so called "radiator function" which corresponds to the shape function known in heavy hadron decays. In this way some new insight into the origin of the shape function is obtained. As a byproduct, a parameterization of the radiatively corrected shape function is suggested which can be implemented in Monte Carlo studies of inclusive heavy hadron decays.
\end{abstract}

\footnotetext{
†From Oct. 1st 2000: Theory Group, CERN, CH-1211 Geneva 23

$\S$ From Oct. 1st 2000: Theory Group, KEK, Tsukuba, Ibaraki, 305-0801, Japan
} 


\section{Introduction}

It has become a standard method to describe inclusive heavy hadron decays in the framework of an expansion in inverse powers of the heavy quark mass $m_{Q}$. This approach allows us to compute lifetimes and branching ratios in a QCD framework, where radiative corrections due to gluon radiation can be systematically computed, while non-perturbative effects can be parameterized to obtain estimates on their size.

This method also yields reliable answers for differential decay rates such as energy spectra or invariant mass spectra that hold in practically all phase space with the exception of certain endpoint regions. These regions are characterized by the kinematic situation in which the heavy quark decays into a practically massless light quark, and all the emitted gluons are collinear to the light quark. In such a situation the hadronic invariant mass in the final state is small while the hadronic energy is still large.

It is well known that in these regions the standard $1 / m_{Q}$ expansion breaks down and has to be replaced by an expansion in twists, very similar to what happens in deep inelastic scattering. The leading twist term has been discussed extensively [1] and is described in terms of a single non-perturbative function, the light cone distribution function of the heavy quark. It has been shown that this approach is in for a certain range of parameters equivalent to the ACCMM model, which is very popular in the phenomenological description of inclusive heavy flavour decays.

However, it is by not trivial to combine the radiative QCD corrections with this light cone distribution function in a consistent way. It has been observed in [2] that one may think of the light cone distribution function as defined in [1] as a convolution of a "soft" and a "jet-like" contribution, where the latter contains all the collinear singularities such as the Sudakov Logarithms.

In the present note we give a practical parameterization of the radiatively

corrected light cone distribution function which can be used to implement the leading radiative and non-perturbative corrections into a decay rate known at tree level. The spirit of this approach is very much along the lines which have been used in high energy $e^{+} e^{-}$or $e p$ processes to implement the leading radiative corrections, e.g. for the $Z_{0}$ line shape [3]. In these processes the the leading logarithmic resummation of the QED radiative corrections leads to a so called "radiator function" which is universal and has to be convoluted with the tree level process to obtain a radiatively corrected cross section. However, in the case of $B$ decays the situation is a little more complicated due to the presence of nonperturbative effects and the Sudakov logarithms, but the physics is essentially the same.

In the next section we discuss the formalism and introduce the parameterization for the analogue of the radiator function for heavy hadron decays. In section 3 we consider a few applications of our formalism, finally we conclude. 


\section{2 "Radiator Functions" in heavy hadron decays}

The situation in inclusive heavy hadron decays is completely analogous to the one in high energy $e^{+} e^{-}-$collisions. In both cases "initial state radiation" diminishes the cms energy of the "partonic" process. While in $e^{+} e^{-}$collisions the initial state radiation is the emission of soft and collinear (to the incident particles) photons, it is the emission of soft and collinear gluons in heavy hadron decays. The soft gluons correspond to a completely non-perturbative function, while the collinear ones lead to Sudakov-like Logarithms, for which also resummation formulae exist.

In high energy $e^{+} e^{-}$processes the bulk of the QED radiative corrections is obtained by a convolution of the "partonic" (i.e. tree level) cross section with a radiator function, such that

$$
d \sigma(s)=\int_{0}^{1} d x_{1} d x_{2} D_{e^{-}}\left(x_{1}, s, m_{e}^{2}\right) D_{e^{+}}\left(x_{2}, s, m_{e}^{2}\right) d \sigma_{t r e e}\left(x_{1} x_{2} s\right)
$$

where the tree-level cross section is taken at a reduced cms energy $x_{1} x_{2} s$. The radiator function $D_{e^{-(+)}}(x)$

$D\left(x, s, m_{e}^{2}\right)=\delta(1-x)+\int_{m_{e}^{2}}^{s} \frac{d k^{2}}{k^{2}} \frac{\alpha\left(k^{2}\right)}{2 \pi} P(x)+\ldots \approx \delta(1-x)+\frac{\alpha}{2 \pi} \ln \left(\frac{s}{m_{e}^{2}}\right) P(x)+\cdots$

with

$$
P(x)=\left(\frac{1+x^{2}}{1-x}\right)_{+}=\frac{1+x^{2}}{1-x}-\delta(1-x)+\lim _{\epsilon \rightarrow 0} \int_{\epsilon}^{1} d z \frac{1+z^{2}}{1-z}
$$

describes the energy loss of the incident particles due to radiation of soft and collinear photons including leading logarithms of the form $\ln \left(s / m_{e}^{2}\right)$. The ellipses denote terms of higher order, which we shall not discuss here. Furthermore, the radiator function depends on the scale of the process $\sqrt{s}$ and on a "regularization scale" $m_{e}$ cutting off the collinear singularities of the photon emission.

While the full radiative corrections (i.e. the ones beyond the leading logs) have to be discussed at the level of amplitudes, the leading logarithmic radiative corrections are described on the level of probabilities: $D(x)$ can be interpreted as the probability that the incident particles loose the energy fraction $x$ due to radiation.

It is well known that this concept is universal and can be applied to the QCD case as well. In deep inelastic scattering the radiator functions become the parton distributions and the scale corresponding to the lower limit of the integration in (2) becomes the factorization scale.

In inclusive heavy hadron decays this works completely analogous. In this case the radiator function is the shape function or the light cone distribution function as given in [1]. The physical picture is the same: The cms-energy available for 
the decay is the mass $M$ of the heavy hadron, but the partonic decay happens at a smaller energy $m_{Q}+k_{+}$, where now $-m_{Q} \leq k_{+} \leq \bar{\Lambda}$ corresponds to the scaling variable $x$ of the radiator function in (2)).

The masses of the hadron $M$ and of the mass $m_{Q}$ of the heavy quark are related to order $1 / m_{Q}$ by

$$
M=m_{Q}+\bar{\Lambda}
$$

The corresponding decay rates in the infinite mass limit are in analogy to (1) [1]

$$
d \Gamma=\int_{-\infty}^{\bar{\Lambda}} d k_{+} R\left(k_{+}, m_{Q}\right) d \Gamma_{\text {tree }}\left(m_{Q}+k_{+}\right)+\mathcal{O}\left(1 / m_{Q}^{2}\right)
$$

where $d \Gamma_{\text {tree }}$ is the tree-level partonic rate and $R\left(k_{+}, \mu\right)$ is the "radiator function" for heavy hadron decays.

It has been argued in [2] that this function can again be decomposed into a "soft" and a "jet-like" part, such that

$$
R\left(k_{+}, \mu\right)=\int_{-\infty}^{\bar{\Lambda}} d l_{+} J\left(k_{+}-l_{+}, \mu\right) f\left(l_{+}, \mu\right)
$$

where $J$ can be computed in perturbation theory and $f$ is a non-perturbative quantity. The factorization into a perturbative part and a non-perturbative contribution involves a factorization scale $\mu$, the dependence on which has been considered in [4, 5].

It is interesting to consider the dependence of this formalism on the on the heavy quark mass; we shall use this information later to adjust the parameters. It is well known that the total rates are proportional to the mass of the heavy quark $m_{Q}$ such that

$$
\Gamma=\gamma m_{Q}^{5}\left(1+b \frac{\alpha_{s}\left(m_{b}\right)}{\pi}+\cdots+\mathcal{O}\left(1 / m_{Q}^{2}\right)\right)
$$

where $b$ is a constant depending on the process under consideration and also on the choice of the mass definition. In fact, using the pole mass in (77) generically yields large and negative coefficients $b$ and it has been argued [6] that a different choice of the mass definition renders $b$ small for all processes. For instance, using instead of the pole mass the $\overline{M S}$ mass and employing the one loop relation

$$
m_{b}^{\text {pole }}=m_{b}^{\overline{M S}}(\mu)\left(1+\frac{\alpha_{s}}{\pi}\left[\frac{4}{3}-\ln \left(\frac{m_{b}^{2}}{\mu^{2}}\right)\right]\right)
$$

the corresponding coefficient for the first-order correction to the rate becomes

$$
b \rightarrow b+\frac{20}{3}-5 \ln \left(\frac{m_{b}^{2}}{\mu^{2}}\right)
$$


On the other hand, we may also compute the total rate in the shape function formalism by integrating (5). Inserting the tree level result for the total rate, we have

$$
\Gamma=\int_{-\infty}^{\bar{\Lambda}} d k_{+} R\left(k_{+}, m_{Q}\right) \gamma m_{Q}^{5}\left(1+5 \frac{k_{+}}{m_{Q}}+\mathcal{O}\left(1 / m_{Q}^{2}\right)\right)
$$

We use the conditions on the shape function

$$
\begin{aligned}
\int_{-\infty}^{\bar{\Lambda}} d k_{+} f\left(k_{+}, \mu\right) & =1 \\
\int_{-\infty}^{\bar{\Lambda}} d k_{+} k_{+} f\left(k_{+}, \mu\right) & =\delta m_{Q}(\mu)
\end{aligned}
$$

Note that the second relation is the equation of motion for the heavy quark, where a possible residual mass term $\delta m_{Q}$ [7], which represents the difference between the mass used in the Lagrangian and the mass used in the definition of the heavy quark momentum $p=m_{Q} v$, has been kept. Obviously, at tree level we recover the total rate $\Gamma=\gamma m_{Q}^{5}$, if we have $\delta m_{Q}=0$

Our aim is to include the large radiative correction coming from the quark mass into a radiatively corrected shape function as defined in (6). This involves the first moment of $R$ defined in (6) and we obtain

$$
\Gamma=\gamma m_{Q}^{5}\left[\left(\int_{-\infty}^{\bar{\Lambda}} d k_{+} R\left(k_{+}, m_{Q}\right)\right)+5\left(\int_{-\infty}^{\bar{\Lambda}} d k_{+} \frac{k_{+}}{m_{Q}} R\left(k_{+}, m_{Q}\right)\right)+\mathcal{O}\left(1 / m_{Q}^{2}\right)\right]
$$

We assume that the non-perturbative function $f$ satisfies the normalization conditions (11,12) at some small scale $\mu$, and we find

$$
\Gamma=\gamma m_{Q}^{5}\left[\left(\int_{-\infty}^{0} d k_{+} J\left(k_{+}, \mu\right)\right)\left(1+5 \frac{\delta m_{Q}}{m_{Q}}\right)+\int_{-\infty}^{0} d k_{+} \frac{k_{+}}{m_{Q}} J\left(k_{+}, \mu\right)+\mathcal{O}\left(1 / m_{Q}^{2}\right)\right]
$$

This shows that the radiative corrections to the total rate are mainly given by the integral over the "jet-like" function. This is a universal function and hence we again find that some large portion of the radiative corrections (namely the leading log contribution) is universal.

However, there also appears a contribution of order $1 / m_{Q}$ which according to (77) has to be zero. This means that the residual mass term has to be chosen in such a way that the corrections of order $1 / m_{Q}$ vanish, i.e.

$$
\delta m_{Q}=-\int_{-\infty}^{0} d k_{+} k_{+} J\left(k_{+}, \mu\right) / \int_{-\infty}^{0} d k_{+} J\left(k_{+}, \mu\right)
$$


Let us now discuss the "jet-like" function explicitely up to order $\alpha_{s}$. The leading logarithmic piece is given by

$$
J\left(k_{+}, \mu\right)=\left(1+\frac{\alpha_{s}}{3 \pi} b_{1}\right) \delta\left(k_{+}\right)-\frac{4 \alpha_{s}}{3 \pi} \Theta\left(-k_{+}\right)\left(\frac{\ln \left(-k_{+} / \mu\right)}{-k_{+}}\right)_{+}
$$

where the double logs $\ln \left(-k_{+} / m_{Q}\right) / k_{+}$are universal, while the single logs are not universal; their size is fixed by an appropriate choice of the scale $\mu$.

Using (16) we can compute the normalization and the first moment for which we get

$$
\begin{aligned}
\int_{-\infty}^{0} d k_{+} J\left(k_{+}, \mu\right) & =1+\frac{\alpha_{s}}{3 \pi} b_{1} \\
\int_{-\infty}^{0} d k_{+} k_{+} J\left(k_{+}, \mu\right) & =\frac{4 \alpha_{s}}{3 \pi} M\left[\ln \left(\frac{M}{\mu}\right)-1\right]
\end{aligned}
$$

where we have inserted $-M$ as a lower cut-off for the $\xi_{+}$integral.

This cut off dependence reflects the fact that although all moments of the non-perturbative function $f$ exist' the moments of the radiatively corrected function $R$ pick up power divergencies. The physical origin of this problem is the "bremsstrahlung" spectrum of the gluon emission behaving as $1 /\left(-k_{+}\right)$and thus the the first moment picks up a linear divergence.

Clearly the physical limit of $k_{+}$is $-m_{Q}$ and thus we shall identify $M=m_{Q}$. The absence of $1 / m_{Q}$ corrections in the total rate thus means that the first moment of the non-perturbative function has to be

$$
\delta m_{Q}=-\frac{4 \alpha_{s}}{3 \pi} m_{Q}\left[\ln \left(\frac{m_{Q}}{\mu}\right)-1\right]
$$

while $b_{1}$ is given by the large and universal correction to the total rate.

Again we consider using a different mass definition in the shape function formalism. For instance, using the $\overline{M S}$ mass defined in (8) we get for the mass difference

$$
m_{b}^{\text {pole }}-m_{b}^{\overline{M S}}(\mu)=m_{b} \frac{\alpha_{s}}{\pi}\left[\frac{4}{3}-\ln \left(\frac{m_{b}^{2}}{\mu^{2}}\right)\right]
$$

which at least for the non-logarithmic terms agrees with $\delta m_{Q}$ computed from the first moment of the shape function. This leads to the conjecture, that the bulk of the radiative corrections at the leading twist level can indeed be absorbed by an appropriate choice of the mass definition, even for differential quantities affected by the shape function. The shape function itself depends on the mass

\footnotetext{
${ }^{1}$ This means that $f$ has to decrease exponentially as $k_{+} \rightarrow-\infty$
} 
definition via its first moment, which has to be shifted by a $\delta m_{Q}$ corresponding to the difference between the mass definition employed and the pole mass.

For practical applications, (16) is still not acceptable, since the "jet-like" function is interpreted in a similar way as in the case of $e^{+} e^{-}$annihilation as a probability of having the tree level process at a reduced cms-energy. However, (16) is not positive definite. This problem has been discussed in [8] where it was shown that sub-leading contributions render the decay rates positive. Thus for phenomenological applications we add terms corresponding to sub-leading pieces. Of course, in general terms of sub-leading twist depend on the specific process, but here we add universal terms which are fixed by comparing to the results of [8]. The formula we are going to use is

$J\left(k_{+}, \mu\right)=\left(1+\frac{\alpha_{s}}{3 \pi} b_{1}\right) \delta\left(k_{+}\right)+\frac{\alpha_{s}}{3 \pi} \Theta\left(-k_{+}\right)\left[-4\left(\frac{\ln \left(-k_{+} / \mu\right)}{-k_{+}}\right)_{+}+b_{2} \frac{k_{+}}{m_{Q}^{2}}+b_{3} \frac{1}{m_{Q}}\right]$

The three constants appearing in (21) have to be determined from some input. One condition is that the large radiative corrections are taken into account, which fixes $b_{1}$ as discussed above. Furthermore, the minimal, kinematically allowed value of $k_{+}$is $-m_{Q}$ where the rate should vanish, thus we require

$$
J\left(k_{+}=-m_{Q}, \mu\right)=0 .
$$

Furthermore, the lower limit of the $k_{+}$integration is now $m_{Q}$.

These conditions can be fulfilled with suitable $b_{1}$ and $b_{3}$ for any choice of $b_{2}$. The coefficient $b_{2}$ multiplies a term of sub-leading twist [8] that could in principle be neglected; for a vanishing $b_{2}$, however, $J\left(k_{+}, \mu\right)$ becomes negative for some $-m_{Q}<k_{+}<0$. Comparison with explicit calculations [8] suggests a value for $b_{2}$ which in turn fixes the constant $b_{3}$. This way we obtain

$$
\begin{aligned}
J\left(k_{+}, \mu\right)= & \left(1-\frac{\alpha_{s}}{3 \pi} b+8 \ln \frac{\mu}{m_{Q}}-4\right) \delta\left(k_{+}\right)+\frac{4 \alpha_{s}}{3 \pi} \Theta\left(-k_{+}\right) \\
& {\left[\left(-\frac{\ln \left(-k_{+} / \mu\right)}{-k_{+}}\right)_{+}+\left(2 \ln \frac{\mu}{m_{Q}}-2\right) \frac{-k_{+}}{m_{Q}^{2}}+\frac{-3 \ln \left(\mu / m_{Q}\right)+2}{m_{Q}}\right] }
\end{aligned}
$$

Here $b$ is from the $\mathcal{O}\left(\alpha_{s}\right)$ correction to the total decay rate (77).

The scale $\mu$ is not fixed by these considerations; explicit next-to-leading order calculations show that $\mu$ is about $0.1 m_{Q} \ldots 0.2 m_{Q}$ and thus is a low scale. Again this is similar to the high-energy $e^{+} e^{-}$case.

To parameterize the non-perturbative contributions we use the model shape function from [9],

$$
f\left(k_{+}\right)=\frac{32}{\pi^{2} \sigma}\left[1-\left(\frac{k_{+}}{\sigma}\right)\right]^{2} \exp \left[-\frac{4}{\pi}\left(1-\left(\frac{k_{+}}{\sigma}\right)\right)^{2}\right] \Theta\left[1-\left(\frac{k_{+}}{\sigma}\right)\right]
$$


This function has vanishing first moment as required at tree level, i.e. $\delta m_{Q}=0$. A non-vanishing first moment as required once radiative corrections are taken into account is achieved by shifting the argument of $f$ by $k_{+} \rightarrow k_{+}+\delta m_{Q}$, where delta $m_{Q}$ is calculated from the first moment of (21).

The radiatively corrected shape function $R\left(k_{+}, \mu\right)$ for a typical $\mu=0.1146 m_{b}$ (as obtained from an explicit calculation of the $\mathcal{O}\left(\alpha_{s}\right)$ radiative corrections to $\left.b \rightarrow u \ell \bar{\nu}_{\ell}\right)$ is shown in figure 1. Since for the decay $B \rightarrow X_{s} \gamma$ the photon energy spectrum is (except for factors) just the shape function itself with the endpoint shifted to $M / 2$, this diagram at the same time shows the spectrum for $B \rightarrow X_{s} \gamma$.

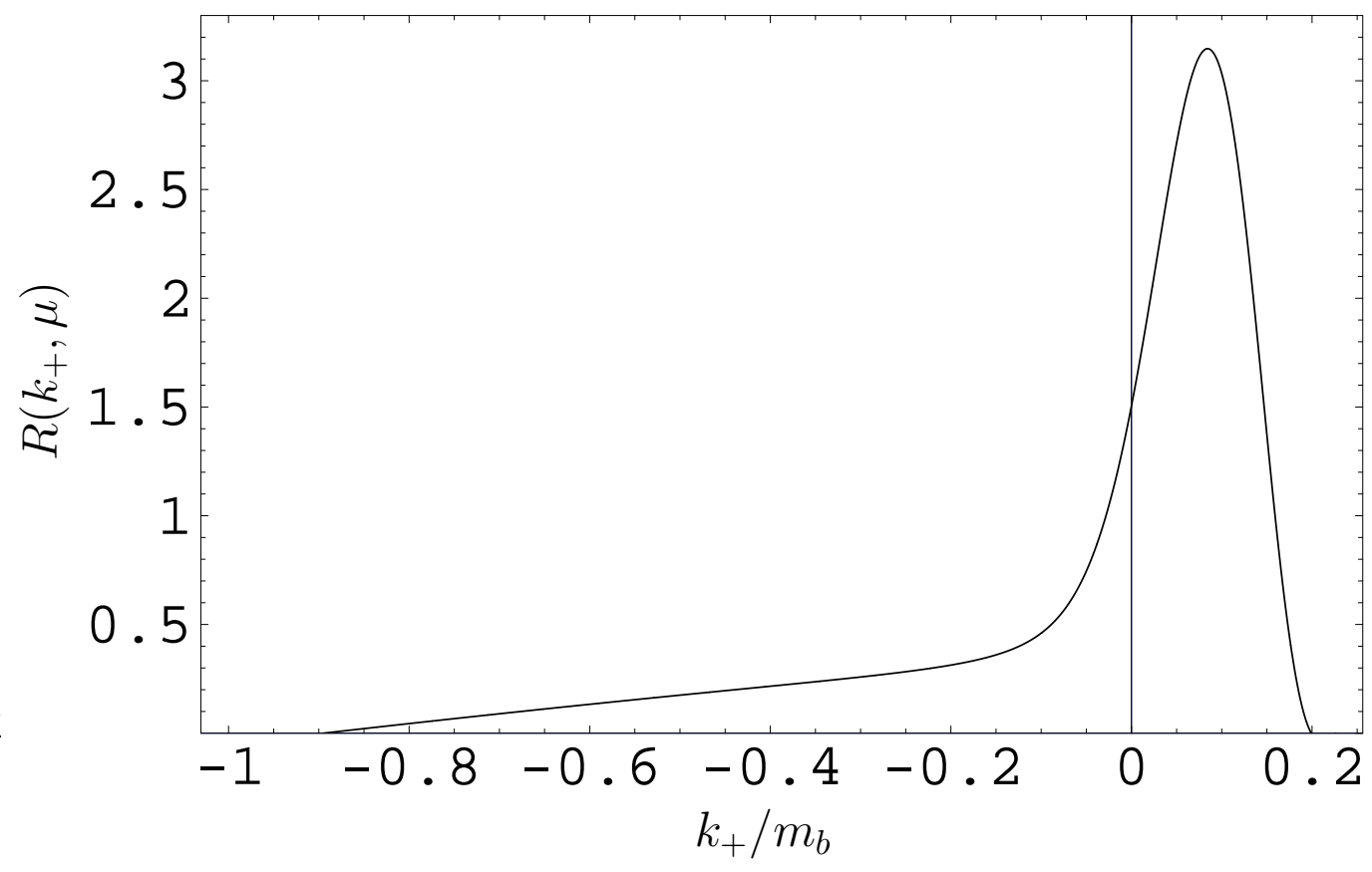

Figure 1: Radiatively corrected shape function $R\left(k_{+}, \mu\right)$.

In the case of the decay $B \rightarrow X_{u} \ell \bar{\nu}$ the spectrum is a step function at maximal lepton energy, which is smoothened by the shape function effect. The corresponding results are shown in figure 2

\section{Applications to the decay $B \rightarrow X_{s} \ell^{+} \ell^{-}$}

As an application we employ the radiatively corrected shape function for the lepton energy spectra in $B \rightarrow X_{s} \ell^{+} \ell^{-}$. While it is well known that the shape functions do not play a significant role in the leptonic invariant mass spectrum, they significantly modify the energy spectra of the leptons. 


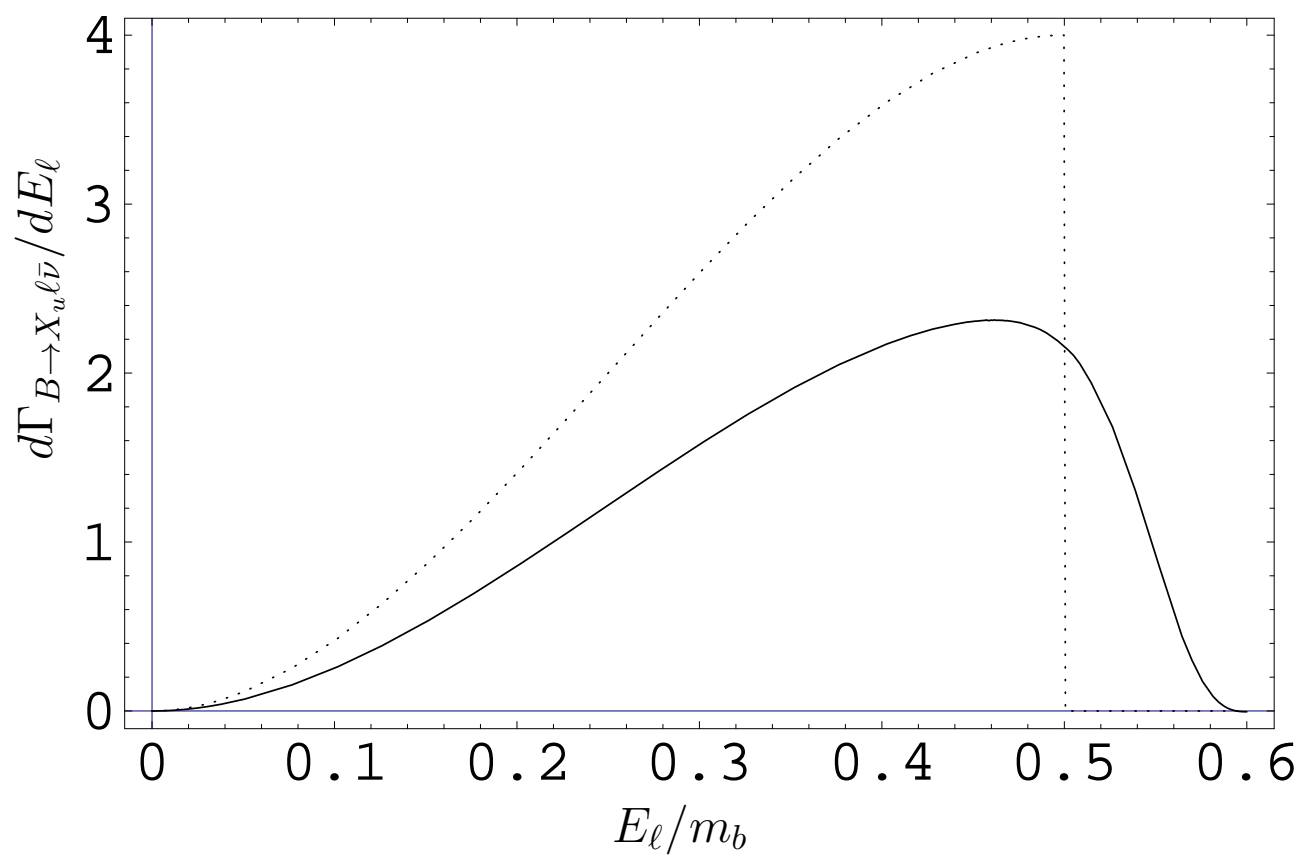

Figure 2: Lepton energy spectrum for $B \rightarrow X_{u} \ell \bar{\nu}$, tree level (dotted line), and including corrections parameterized with the radiator function formalism (solid line). The rate is given in units of $G_{F}^{2} m_{b}^{5}\left|V_{u b}^{2}\right| / 96 \pi^{3}$

Of particular interest are the lepton energy spectra, where the flavour of the decaying $B$ meson has been tagged. These spectra have been suggested to test the coefficients $C_{9}$ and $C_{10}$ of the effective weak Hamiltonian governing weak decays of $b$ quarks. In addition, for experimental reasons an energy cut has been applied to the second lepton.

The partonic spectra show pronounced, step function like structures which will be affected strongly by the shape function effects. In figure (3) we show the $E_{\ell^{+}}$spectrum with a cut of $0.2 m_{b}$ on the energy of the negatively charged lepton both with and without shape function effects. It can be seen how the radiatively corrected shape function not only shifts the endpoint of the spectrum and adjusts the rate but also smears out the features of the partonic spectrum.

\section{Conclusions}

The question of how to implement non-perturbative contributions due to bound state effects in combination with a consistent treatment of radiative corrections has been discussed form a new point of view. Comparing the situation in $B$ decays to the case of $e^{+} e^{-}$annihilation we look at the shape function as the analogue of the radiator function in high energy $e^{+} e^{-}$processes. In both cases the bulk 


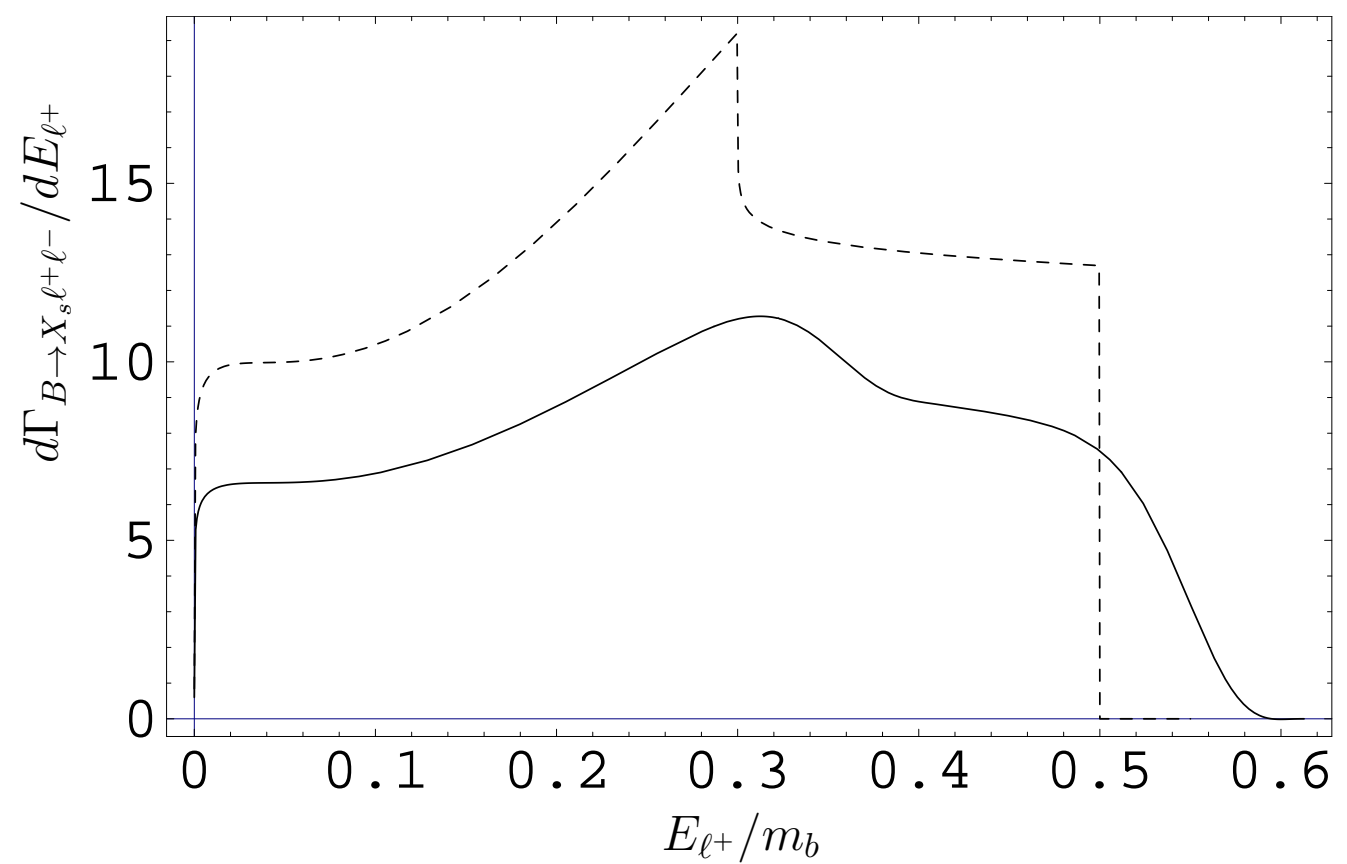

Figure 3: Spectra for the energy of the positively charged lepton in $B \rightarrow X_{s} \ell^{+} \ell^{-}$ with (solid) and without (dashed) shape function corrections. Both spectra include a cut of $0.2 m_{b}$ on the energy of the negatively charged lepton. The spectra are given in units of $G_{F}^{2} \alpha^{2}\left|V_{t s}^{*} V_{t b}\right|^{2} m_{b}^{5} / 3(2 \pi)^{5}$ Standard values have been used for the Wilson coefficients; all our plots use $\alpha_{s}\left(m_{b}\right)=0.215$, and the shape function parameter is $\sigma / m_{b}=0.57 / 4.71$.

of the radiative corrections (and in $B$ decays the main non-perturbative effects) can be accounted for by a convolution of the tree level rate with an appropriate function, which can be interpreted as a probability for a certain energy loss. Since this works at the level of probabilities, this approach can be easily implemented into Monte Carlo programs, which has been done quite successfully for the case of high energy $e^{+} e^{-}$processes [10].

For the case of $B$ decays the situation is complicated by the presence of nonperturbative effects. These have to be parameterized in terms of a so called shape function, which then is combined with the leading radiative corrections into the analogue of the radiator function.

The energy available in the $B$ decay process depends on the mass of the heavy quark in the initial state. On the other hand, the $b$ quark mass is not a physical quantity and thus one has to consider the dependence on the mass definition. It turns out that the main part of the radiative corrections can indeed be absorbed into an appropriate mass definition; this fact is well known for the total rates, but it seems to be true also for the leading twist contributions parameterized by 
the shape function.

We have given a parameterization of the radiatively corrected shape function which includes some sub-leading twist contributions which have been fitted in such a way that the explicitely known decays of the heavy-to-light type are well reproduced. This parameterization may be useful for practical applications, in particular for Monte Carlo simulations of $B$ decays.

\section{Acknowledgments}

SR and TM acknowledge the support of the DFG Graduiertenkolleg "Elementarteilchenphysik an Beschleunigern"; TM acknowledges the support of the DFG Forschergruppe "Quantenfeldtheorie, Computeralgebra und MonteCarlo Simulationen".

\section{References}

[1] M. Neubert, Phys. Rev. D 49, 3392 (1994); M. Neubert, Phys. Rev. D 49, 4623 (1994); T. Mannel and M. Neubert, Phys. Rev. D 50, 2037 (1994); I. I. Bigi, M. A. Shifman, N. G. Uraltsev, and A. I. Vainshtein, Int. J. Mod. Phys. A 9, 2467 (1994).

[2] G. P. Korchemsky and G. Sterman, Phys. Lett. B 340, 96 (1994)

[3] see e.g. O. Nicrosini and L. Trentadue in Radiative Corrections to $e^{+} e^{-}$ Collisions, Edited by J.H. Kühn, Springer-Verlag, 1989

[4] C. Balzereit, T. Mannel and W. Kilian, Phys. Rev. D 58, 114029 (1998)

[5] U. Aglietti, "The shape function in field theory," CERN-TH/2000-278, hepph/0009214; U. Aglietti and G. Ricciardi, "The structure function of semiinclusive heavy flavour decays in field theory," CERN-TH-2000-071, DSF-T7-00, hep-ph/0003146.

[6] I. I. Bigi, M. A. Shifman, N. G. Uraltsev, and A. I. Vainshtein in [1]

[7] A. F. Falk, M. Neubert and M. Luke, Nucl. Phys. B 388, 363 (1992)

[8] T. Mannel and S. Recksiegel, Phys. Rev. D 60 (1999) 114040

[9] T. Mannel and M. Neubert in [1]

[10] see e.g. H. Anlauf, H. D. Dahmen, A. Himmler, P. Manakos, T. Mannel and T. Ohl, Nucl. Phys. Proc. Suppl. 37 B, 81 (1994); S. Jadach, B. F. Ward and Z. Was, Nucl. Phys. Proc. Suppl. 89, 106 (2000) 